\title{
COMUNICACIÓN EDUCATIVA Y MEDIACIONES TECNOLÓGICAS: HACIA NUEVOS AMBIENTES \\ DE APRENDIZAJE
}

Crovi Druetta, Delia

Ed. ILCE, Serie Autores y Actores de la Educación, México, 2007, 71 p.

\section{DATOS DE LA AUTORA}

Es profesora e investigadora de la Facultad de Ciencias Políticas y Sociales de la Universidad Nacional Autónoma de México. Colaboradora del Instituto Latinoamericano de la Comunicación Educativa. Coordinadora del grupo de trabajo de Comunicación y Educación de la Asociación Latinoamericana de Investigadores de la Comunicación.

\section{CONTENIDO}

La elección de esta obra responde al interés de mostrar las posibilidades de la educación en el siglo XXI, a partir del uso de la tecnología.

El texto recorre las diferentes generaciones tecnológicas y su repercusión en los procesos de enseñanza-aprendizaje, principalmente en el ámbito de la educación a distancia. En la parte inicial, la autora muestra las repercusiones del uso de medios como la correspondencia tradicional y los mass media en la educación, para dar paso en la parte final a los proceso de virtualidad en los nuevos ambientes de aprendizaje. 
Es interesante cómo, al final de la obra, la autora enumera los cambios que ha experimentado la educación del siglo XXI, partiendo de dicho recorrido histórico.

La reflexión del libro gira en torno a los procesos educativos desde el enfoque de la comunicación; la autora concibe a esta última como la base de todo proceso de enseñanza.

La intención de Delia Crovi es analizar los sistemas educativos que se apoyan en el uso de la tecnología, para lo cual se mencionan las distintas generaciones tecnológicas que han impactado en la enseñanza (p. 10).

El camino que sigue la autora para reflexionar sobre el tema es: primero, hacer referencia a la comunicación educativa, proceso básico de enseñanza; en segundo lugar, presentar desde una perspectiva histórica los momentos por los que ha pasado el proceso de enseñanzaaprendizaje (PEA) a partir de la influjo de las diferentes tecnologías, y finalmente analiza el PEA influenciado por la intervención de las tecnologías de la información y la comunicación.

Así, en la primera parte se presenta la relación entre educación y comunicación desde tres perspectivas: la comunicación en la educación, la educación para la recepción, y la educación en red. «[...] Es decir, aquella que se imparte con el auxilio de las computadoras conectadas a Internet, la red de redes más importante de la actualidad» (p. 11). El énfasis de la obra está en la última.

En este mismo apartado se rescata el tema del diálogo como base de la comunicación educativa, y se presentan tres principios comunes a la educación y la comunicación que la autora retoma de Francisco Sierra en el libro Introducción a la teoría de la comunicación educativa. Dichos principios son: relacionabilidad, alteridad y diálogo.

La tecnología ha influido tanto en los ambientes educativos presenciales como en los ambientes educativos a distancia. Sin embargo, por ser más evidente en los últimos, Delia Crovi enfatiza la influencia de las 
generaciones tecnológicas en éstos; por ello, en el apartado dos del libro aquí reseñado se especifican los tres momentos en los cuales la tecnología influye en la educación a distancia.

El primero hace referencia a la tecnología de la correspondencia. «Durante la segunda década del siglo XX, muchos jóvenes latinoamericanos que vivían en lugares apartados de las grandes urbes, comenzaron a interesarse por un nuevo tipo de oferta educativa: cursos a distancia por correspondencia [...]» (p. 21). Años después, la correspondencia fue sustituida por los sistemas de paquetería.

Un segundo momento surge con el uso de los medios masivos - específicamente de la televisión-, ejemplo de ello es la tele-secundaria en México.

Según la autora, el modelo de tele-secundaria ha pasado por tres grandes períodos: el primero basado en la tele-clase en donde un actor/profesor impartía su cátedra y ésta se trasmitía en vivo; el segundo, donde las tele-clases se sustituyeron por programas grabados con los cuales se apoyaba la labor docente en el aula, y el tercer período en que los programas se estructuran en torno a segmentos cortos y se utilizan otros medios como las computadoras (p. 28).

El tercer momento se relaciona con la instrucción programada y la tecnología educativa en la cual se unen los componentes del tecnólogo, la computadora y el conductismo como concepción de la educación. La autora destaca que en esa etapa también se empieza a trabajar con la integración de medios para crear contenidos educativos.

Después de presentar un recorrido histórico sobre el uso de medios en la educación a distancia, Delia Crovi analiza el tema de los nuevos ambientes de aprendizaje en el tercer apartado. En éste, acota: «Hoy en día es más adecuado hablar de educación en red, educación virtual o de nuevos ambientes de aprendizaje, porque son conceptos que permiten inferir la complejidad de elementos que intervienen en la educación mediada por las redes» (p. 35). 
Crovi, especialista en temas de comunicación y educación mediada por tecnología, afirma que el concepto de nuevos ambientes de aprendizaje está en construcción y se ha llegado a él por la necesidad de explicar una manera diferente de organizar el aprendizaje a partir del uso de las tecnologías de la información y la comunicación.

Los nuevos ambientes de aprendizaje crean una situación educativa en la cual el alumno necesita desarrollar pensamiento creativo, autoaprendizaje y trabajo colaborativo, mientras que el profesor desempeña el papel de orientador y tutor, todo ello auxiliado por la tecnología. Por lo anterior, la autora señala: «[...] los elementos que se ponderan en la creación de un nuevo ambiente de aprendizaje varían según situaciones y recursos, ya que el problema no se limita sólo a introducir tecnologías» (p. 35).

Entonces, ¿cuál es la novedad de estos ambientes de aprendizaje? La autora responde que ésta radica en que los nuevos ambientes de aprendizaje atienden a expectativas diversas y ello abre grandes oportunidades educativas.

La autora presenta como punto de partida de los nuevos ambientes de aprendizaje, los ejes de la educación superior propuestos por la UNESCO: pertinencia, calidad, administración-financiación y cooperación. En donde las TIC ocupan un lugar destacado como instrumentos de cambio (p. 37).

Crovi continúa el tercer apartado reflexionando sobre las características del tipo de enseñanza que ha propiciado la emergencia de los nuevos ambientes de aprendizaje mediados por las tecnologías de la información. Dichos ambientes son un espacio social el cual ha recibido diversos nombres como: ciberespacio, telépolis, ciberredes, carreteras de la información y cibercultura.

Los anteriores han generado una idea de la virtualidad en las relaciones, por ello Delia Crovi dedica un espacio para explicar el concepto de virtualidad, retomando las ideas de Pierre Lévy en el libro ¿Qué es lo virtual? y concluyendo: «[...] virtual es aquello que produce un efecto, pero 
no lo hace de presente, lo que nos acerca al campo imaginario, de la realidad simbólica, o sea, de la representación de lo real o de los conceptos a través de símbolos» (p. 41).

Retomando lo anterior, el interés de la autora es reflexionar acerca de lo virtual como producto de las nuevas tecnologías y, en especial, de las redes como Internet. «[...] la educación en red, virtual, tiene su centro de interés en la organización, en el correcto flujo de información de relaciones, así como de actividades entre los actores del proceso educativo, debido a que existe un rompimiento de los conceptos tradicionales de tiempo y espacio» (p. 45).

La creación de espacios virtuales genera ciudadanos virtuales, es decir disponibles, en línea abierta las veinticuatro horas del día para recibir y enviar mensajes. El sentido de privacidad cambia porque las personas están dispuestas a exponerse a la irrupción de los otros, tratando temas referentes a los diversos ámbitos de la vida de un ser humano: ocio, trabajo y educación.

Así, las nociones de tiempo y espacio cambian a partir del uso de las tecnologías de la información y la comunicación, específicamente de las redes.

Finalmente con un estilo sencillo, en el apartado cuarto del texto, Crovi formula unas reflexiones a manera de cierre, destacando que la base del cambio en los sistemas educativos radica en los modelos económicos y ejemplifica: ¿cómo el taylorismo y la sociedad industrial influyeron en la propuesta de educación a distancia de la época?

El planteamiento del libro resulta especialmente interesante para las personas que deseen conocer acerca de la generación de los nuevos ambientes de aprendizaje en la era de la información.

El libro Comunicación educativa y mediaciones tecnológicas: hacia nuevos ambientes de aprendizaje, realiza una aportación valiosa, al igual que la de otros autores especialistas en el tema, al presentar los escenarios político-económicos a la par del desarrollo tecnológico, 
como las condiciones que propician el cambio crucial en los modelos de educación a distancia.

Además, son de gran valor las siete reflexiones finales sobre las cuales la autora invita a trabajar desde el campo de la investigación y la práctica educativa: las posibilidades de orden técnico, vistas desde la comunicación educativa; el desplazamiento de la atención en el PEA hacia el alumno; el aprendizaje para toda la vida; el aprovechamiento de las nuevas condiciones tecnológicas; los sistemas de certificación de la educación; el financiamiento de la educación y la tendencia creciente a la globalización educativa.

A manera de cierre podemos afirmar que es un buen libro que puede recomendarse al estudioso de la educación mediada por las tecnologías de la información y la comunicación, específicamente a través de las redes y lo que la autora denomina «convergencia tecnológica».

Claudia F. Ortega Barba 